\title{
Effects of intermittent fasting on liver physiology and metabolism in mice
}

\author{
JIANBO MA ${ }^{1}$, YAN CHENG ${ }^{1,2}$, QIANG SU $^{1}$, WEN AI $^{3}$, LING GONG $^{4}$, YUEYING WANG ${ }^{1}$, \\ LINHAO LI ${ }^{1}$, ZHONGREN MA ${ }^{1}$, QIUWEI PAN ${ }^{1}$, ZILIN QIAO ${ }^{*}$ and KAN CHEN ${ }^{1,5^{*}}$ \\ ${ }^{1}$ Key Laboratory of Biotechnology and Bioengineering of State Ethnic Affairs Commission, Biomedical Research Center, \\ Northwest Minzu University; ${ }^{2}$ Experimental Center, Northwest Minzu University, Lanzhou, Gansu 730030; \\ ${ }^{3}$ Department of Cardiology, Union Shenzhen Hospital, Huazhong University of Science and Technology, Shenzhen, \\ Guangdong 518102; ${ }^{4}$ Department of Liver Diseases, The Affiliated Hospital of Hangzhou Normal University, \\ Hangzhou, Zhejiang 310015; ${ }^{5}$ College of Life Science and Medicine, Zhejiang Sci-Tech University, \\ Hangzhou, Zhejiang 310018, P.R. China
}

Received February 26, 2020; Accepted June 8, 2021

DOI: $10.3892 /$ etm.2021.10382

\begin{abstract}
A broad spectrum of health benefits from intermittent fasting have been reported in studies on animal models and human subjects. However, the underlying mechanisms of these beneficial effects remain largely elusive. The present study aimed to explore the effects and potential mode of action of intermittent fasting in mouse models with a focus on the liver. C57BL/6 mice were subjected to intermittent fasting or ad libitum feeding as controls. It was determined that $12 \mathrm{~h}$ of daily intermittent fasting for 30 days significantly reduced the cumulative food intake compared with that in mice with ad libitum feeding. Fasting resulted in a significantly reduced
\end{abstract}

Correspondence to: Dr Kan Chen or Mr. Zilin Qiao, Key Laboratory of Biotechnology and Bioengineering of State Ethnic Affairs Commission, Biomedical Research Center, Northwest Minzu University, 1 Northwest New Village, Lanzhou, Gansu 730030, P.R. China

E-mail: chenkan@zstu.edu.cn

E-mail: qiaozilin@xbmu.edu.cn

${ }^{*}$ Contributed equally

Abbreviations: AL, ad libitum; ALP, alkaline phosphatase; ALT, alanine aminotransferase; ALB, albumin; AMP, adenosine monophosphate; AST, aspartate aminotransferase; AST/ALT, aspartate aminotransferase/alanine aminotransferase; A/G, albumin/globulin; ATP, adenosine triphosphate; FMN, flavin mononucleotide; GDP, guanosine diphosphate; GLO, globulin; $\gamma$-GT, $\gamma$-glutamyl transpeptidase; IF, intermittent fasting; IF\&AL, IF followed by AL; LDH, lactate dehydrogenase; NADP, nicotinamide adenine dinucleotide phosphate; PEP, phosphoenolpyruvate; $\mathrm{TP}$, total protein; TPP, thiamine pyrophosphate

Key words: intermittent fasting, mouse model, bodyweight, liver mass, liver metabolism liver mass but only had a minimal effect on bodyweight. The effects on the liver by 30 days of fasting were not reversed by subsequent ad libitum refeeding for 30 days. Among the measured blood biochemical parameters, the levels of blood glucose were decreased, while the levels of alkaline phosphatase were increased in fasting mice. Of note, targeted metabolic profiling revealed global elevation of metabolites in the livers of fasting mice. These metabolic molecules included adenosine triphosphate, nicotinamide adenine dinucleotide phosphate (NADP), reduced NADP and succinate, which are essentially involved in the citric acid cycle and oxidative phosphorylation. Thus, it was concluded that daily $12 \mathrm{~h}$ of intermittent fasting for one month significantly reduced the liver weight of mice, which is associated with enhanced liver metabolism.

\section{Introduction}

Calorie restriction has been demonstrated to mitigate the age-associated decline of several pathophysiological parameters and to extend the maximum lifespan in various animal species (1-3). Fasting is one type of calorie restriction and has been widely practiced as a type of medical application or religious ritual. Fasting is defined as abstinence from or reduction of food, drink or both for a period typically lasting between $12 \mathrm{~h}$ and 3 weeks, in short-term, long-term or intermittent patterns $(4,5)$. Intermittent fasting is an umbrella term for various diets comprising a cycle of a period of fasting and non-fasting $(6,7)$. In fact, intermittent fasting has been practiced by the Muslim population for over a thousand years in the month of Ramadan. This usually involves 12-16 h of daily fasting by abstinence of both drink and food for one month (8).

The health benefits of intermittent fasting have been extensively demonstrated in animal models (9-11). Furthermore, certain observational studies have been performed suggesting potential benefits of reduced cancer risk and metabolic disease associated with intermittent fasting in humans $(12,13)$. However, the mechanisms of health promotion by fasting have remained largely elusive and metabolic regulation is conceivably 
essential. As a state of negative energy balance, even a single fasting interval in humans (e.g., overnight) may reduce basal concentrations of specific metabolic biomarkers (insulin and glucose) that are associated with chronic diseases (7). It has been indicated that long-term dietary restriction reduces the metabolic rate and bodyweight $(14,15)$, while certain studies reported that fasting improves the metabolic state due to weight loss and a greater extent of fat burning $(16,17)$. In the context of Ramadan fasting, changes may range from a reduction to a rise in bodyweight (18-20).

The liver is a central organ required for metabolic homeostasis $(21,22)$. The liver takes up glucose and synthesizes glycogen and triglycerides following food intake, releases glucose produced by glycogenolysis or gluconeogenesis and triggers ketogenesis during fasting (23). High glucagon is produced during fasting, which stimulates glucose release from the liver to provide a continuous fuel supply for peripheral tissues $(24,25)$. Therefore, observing the alterations of small molecules by metabolic profiling in the liver allows comprehensive analysis of changes in several metabolic and signaling pathways and their interactions $(26,27)$. Previous studies had described the health benefits of intermittent fasting $(7,28,29)$, but little was known about the mechanism-of-action. Furthermore, variety types of 'intermittent fasting' had been studied, but many were not relevant to human population.

It is impossible to get blood samples and liver tissues from healthy volunteers during Ramadan fasting. The present study mimicked the Ramadan fasting pattern in mouse models, aiming to evaluate the effects of daily 12-h intermittent fasting for 1-2 months in mice. The focus of the experiments was on the effects and mode of action of 12-h intermittent fasting on liver physiology and metabolism. These are very relevant to the human population and will add new knowledge to the field.

\section{Materials and methods}

Animal model and treatment regimens. Male C57BL/6 mice (4-6 week-old; $n=51$ ) were purchased from the Experimental Animal Center of Lanzhou Veterinary Research Institute (Lanzhou, China). The animal experiments were approved by the Laboratory Animal Ethics Committee of Northwest Minzu University (Lanzhou, China), which is under the supervision of the Experimental Animal Committee of Lanzhou Veterinary Research Institute. Mice were housed individually in isolated cages (one mouse per cage) at a standardized temperature $\left(18-23^{\circ} \mathrm{C}\right)$ with $\sim 50 \%$ humidity and a 12 -h light/dark cycle. The mice were fed a standard diet (62\% carbohydrate, $26 \%$ protein and $12 \%$ fat) and had free access to purified water. In addition, the health status of the mice was monitored during daily feeding. Excessive weight loss (20\% of the average body weight of mice in the same period), loss of appetite, weakness (inability to eat or drink on their own, inability to stand or barely able stand for $24 \mathrm{~h}$ ) and seizures (30) were considered humane endpoints, which did not happen in subsequent experiments.

In general, the mice were subjected to overnight fasting prior to sacrifice. However, the present study aimed to specifically investigate the effects of intermittent fasting. Therefore, prior to sacrifice, the intermittent fasting group was subjected to $12 \mathrm{~h}$ of fasting, while the control group was maintained with ad libitum feeding. For the 30-day experiment, the mice were randomly divided into two groups ( $\mathrm{n}=10 /$ group) as follows: The ad libitum group (AL) and intermittent fasting group (IF; Fig. 1A). In the 60-day experiment, the mice were randomly divided into three groups ( $n=8-14$ /group), including the AL group, IF group and the intermittent fasting followed by ad libitum feeding group (IF\&AL; Fig. 1B). In the AL group, the mice had free access to food and water, while in the IF group, food and water were removed for $12 \mathrm{~h}$ during the nighttime (from 8:00 p.m. to 8:00 a.m.) on a daily basis (Fig. 1C) $(31,32)$. The food intake and bodyweight of the mice were measured every 10 days until the end of the study. All animal food was purchased from the Double Lion Experimental Animal Feed Technology Co., Ltd. The mice were anesthetized using $4 \%$ isoflurane United States Pharmacopoeia (USP) $100 \%$ (1,000 ml/min; 4\% isoflurane for induction; $500 \mathrm{ml} / \mathrm{min} ; 1.75-2.5 \%$ isoflurane for maintenance) and then sacrificed by cervical dislocation. The livers were immediately isolated and weighed. One portion of the liver was fixed in $4 \%$ paraformaldehyde and the remaining liver was rapidly frozen in liquid nitrogen and then transferred to a $-80^{\circ} \mathrm{C}$ freezer.

Histological examination of mouse liver. The livers were processed in a series of stages, including alcohol dehydration, clearing by xylene and then embedding in paraffin. Deparaffinized sections of the liver $(4 \mu \mathrm{m})$ were rehydrated and stained with $\mathrm{H} \& \mathrm{E}$ according to standard procedures.

Targeted metabolomics by liquid-chromatography tandem mass spectrometry (LC-MS/MS). The 30-day AL and 30-day IF groups were analyzed by targeted metabolomics. Prior to sacrifice, the animals in the IF group were subjected to 12-h fasting, while the control group was able to feed ad libitum. The livers were immediately isolated and rapidly stored in liquid nitrogen after sacrifice and were finally transferred to a $-80^{\circ} \mathrm{C}$ refrigerator. The frozen liver tissues were added in cold methanol/acetonitrile/ $\mathrm{H}_{2} \mathrm{O}$ and vortexed. The sample was homogenized by MP Fastprep-24 Automated Homogenizer (MP Biomedicals) and sonicated by an Ultrasonic Liquid Processors (Scientz JY92-II, Ningbo Scientz Biotechnology Co., Ltd.) in an ice-water bath (30 min each time, 2 times in total), and the mixture was centrifuged for $20 \mathrm{~min}(14,000 \mathrm{x} \mathrm{g}$, $\left.4^{\circ} \mathrm{C}\right)$. Subsequently, the supernatant was dried in a vacuum centrifuge, re-dissolved in acetonitrile/water $(1: 1, \mathrm{v} / \mathrm{v})$ and adequately vortexed, and centrifuged for $20 \mathrm{~min}(14,000 \mathrm{x}$, $\left.4^{\circ} \mathrm{C}\right)$. The final supernatants were collected for LC-MS/MS analysis.

The LC-MS/MS analyses were performed using an UHPLC (1290 Infinity LC; Agilent Technologies, Inc.) coupled with a QTRAP 5500 apparatus (AB Sciex LLC). For Hydrop Interaction Liquid Chromatography separation, the samples were analyzed using an ACQUITY UPLC BEH Amide column (Waters MS Technologies). The Quality Control sample was set for each interval of a certain number of experimental samples in the sample queue to detect and evaluate the stability and repeatability of the system. In electron spray ionization negative mode, the conditions were set as follows: Source temperature, $450^{\circ} \mathrm{C}$; Ion Source Gas1, 45; Ion 
Source Gas2, 45; Curtain gas, 30; IonSapary Voltage Floating, 4,500 V; and MS/MS Analysis mode of detection, ion pair.

Measurement of biochemical markers in blood samples. Peripheral blood samples from the 30-day AL group and 30-day IF group were collected to measure specific biochemical markers. At the time of sample collection, the IF mice had fasted for $12 \mathrm{~h}$, while the control group had ad libitum access to food. Initially, the mice were anesthetized with isoflurane USP $100 \%(1,000 \mathrm{ml} / \mathrm{min} ; 4 \%$ isoflurane for induction; $500 \mathrm{ml} / \mathrm{min} ; 1.75-2.5 \%$ isoflurane for maintenance) and subsequently, peripheral blood was immediately collected from the orbital venous sinus. At least $300 \mu 1$ of blood (maximum $400 \mu \mathrm{l}$ ) was collected from each mouse. The mice were sacrificed immediately by cervical dislocation after blood collection. Mortality was further confirmed by determining the cessation of respiratory and heart function. The serum samples of the mice were harvested by centrifugation (440 x g for $10 \mathrm{~min}$ at room temperature) and stored at $-20^{\circ} \mathrm{C}$ until further analysis. Lilai Biological Co. was commissioned to measure biochemical markers in the blood samples. The concentration levels of glucose, total protein (TP), albumin (ALB) and globulin (GLO), as well as the activity levels of alanine aminotransferase (ALT), aspartate aminotransferase (AST), alkaline phosphatase (ALP), lactate dehydrogenase (LDH) and $\gamma$-glutamyl transferase $(\gamma$-GT) were measured using an auto-analyzer (Beckman LX-20; Beckman Coulter, Inc.).

Statistical analysis. Values were expressed as the mean \pm standard error of the mean (SEM). Comparisons were performed with the Mann-Whitney U-test between two groups or with the Kruskal-Wallis test with Dunn's post-hoc multiple comparisons test. $\mathrm{P}<0.05$ was considered to indicate a statistically significant difference.

Hierarchical clustering analysis was performed using cluster 3.0 (http://bonsai.hgc.jp/ mdehoon/software/cluster/software.htm) and the Java Treeview 3.0 software (http://jtreeview.sourceforge.net). The principal component analysis was performed using MetaboAnalyst 4.0 software. The Euclidean distance algorithm for similarity measurement and the average linkage clustering algorithm for clustering were selected. The heatmap was used as a visual aid apart from the dendrogram.

\section{Results}

Effects of intermittent fasting on food intake and bodyweight of mice. During the 30-day experiment, a significant increase in the bodyweight of the mice was noted. The bodyweight exhibited no significant differences between the AL and the IF at the end of the experiment (AL vs. IF, 21.701 \pm 1.305 vs. $21.610 \pm 1.187$, respectively; mean $\pm \mathrm{SEM} ; \mathrm{n}=10$ ). However, the cumulative food intake of AL mice was higher compared with that of the IF mice. To confirm this result, the duration of fasting was extended to 60 days. A total of three groups were included in the 60-day experiment as follows: The AL, IF and IF\&AL (refeeding) groups. The IF\&AL group was subjected to 30-day intermittent fasting followed by 30 days of ad libitum access to food. The bodyweight of the mice in these three groups exhibited no significant difference by the end of the experiment (AL vs. IF vs. IF\&AL, 26.193 \pm 1.680 vs. $26.844 \pm 1.136$ vs. $26.457 \pm 1.779$, respectively; mean \pm SEM; $\mathrm{n}=8-14)$. The bodyweight and food intakes of three different time points were provided in Table I. Overall, the data indicated that 12-h nighttime intermittent fasting did not affect the bodyweight of mice, although it resulted in less cumulative food intake. The bodyweights of the mice subjected to 60-day fasting were significantly increased compared with those at 30 days (30-day AL vs. 60 -day AL, 21.701 \pm 1.305 vs. $26.193 \pm 1.680$, respectively; and 30 -day IF vs. 60-day IF, $21.610 \pm 1.187$ vs. $26.844 \pm 1.136$, respectively; mean \pm SEM), while their weight gain was significantly decreased. This may be explained by their food intake, since almost all of the mice in the 60-day experiments consumed less food than the 30-day group. A comparison of the bodyweight and food intakes of three different time points between the 60- and 30-day IF groups was provided in Table II. The maximum percentage of weight loss (before and after fasting) was 7.07\%.

Reduction in liver weight by intermittent fasting. The livers were isolated, observed and weighed following animal sacrifice. The livers of IF mice appeared smaller than those of the mice in the AL group (Fig. 1D and F). Subsequently, the wet liver weight was determined and the liver weight/total bodyweight (LW/TBW) ratio was determined. The results indicated that the liver mass of IF mice was significantly lower than that of AL mice (Fig. 1E and G) and the LW/TBW ratio of the mice in the IF group was considerably lower than that of the AL group (Fig. 1H and I). Ad libitum refeeding for 30 days did not recover fasting-induced loss of liver mass. The liver weight of the mice subjected to 60-day intermittent fasting was significantly increased compared with that of the 30-day IF mice, while the LW/TBW ratio was not significantly altered (Fig. 1J and K). This suggested that prolonged intermittent fasting did not alter the effects noted previously.

Subsequently, the morphological changes in the mouse liver tissues were examined. Histological assessment of the liver tissues by H\&E staining indicated that the hepatocyte plates were well developed and that the sinusoids were clearly visible in all of the livers (Fig. 2A and B).

Intermittent fasting alters the levels of liver-associated biochemical markers in blood samples from mice. Subsequently, the blood biochemical markers associated with liver physiology or function were assessed. The concentration levels of glucose, TP, ALB and GLO and the activity levels of ALT, AST, ALP, LDH and $\gamma$-GT were measured. The blood glucose levels were significantly decreased in IF mice compared with those in AL mice (IF vs. AL, $1.391 \pm 0.914$ vs. $5.726 \pm 0.648$, respectively; mean \pm SEM; $n=7 ; \mathrm{P}<0.01$; Fig. $3 \mathrm{~A}$ ). The activity levels of ALP were significantly increased in IF mice (IF vs. AL, $171.571 \pm 13.465$ vs. $152.829 \pm 17.130$, respectively; mean $\pm \mathrm{SEM}$; $\mathrm{n}=7$; $\mathrm{P}<0.05$; Fig. $3 \mathrm{~B})$. No significant change was observed for the other parameters tested (Fig. 3C-K). These results suggested that intermittent fasting did not affect the liver function of mice, whereas it was likely to regulate metabolism.

Intermittent fasting rewires liver metabolism. To finally assess the effects of intermittent fasting on liver metabolism, targeted 
A

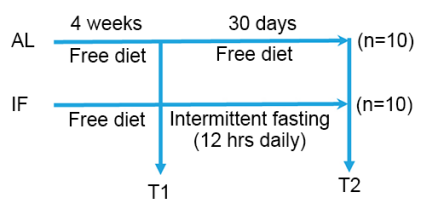

B

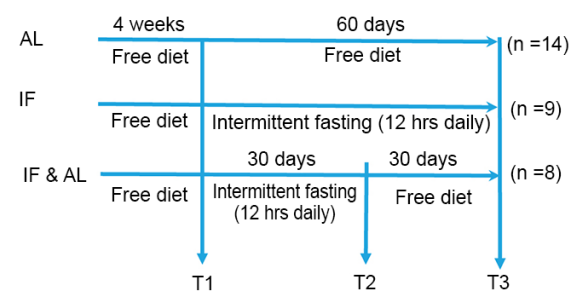

D

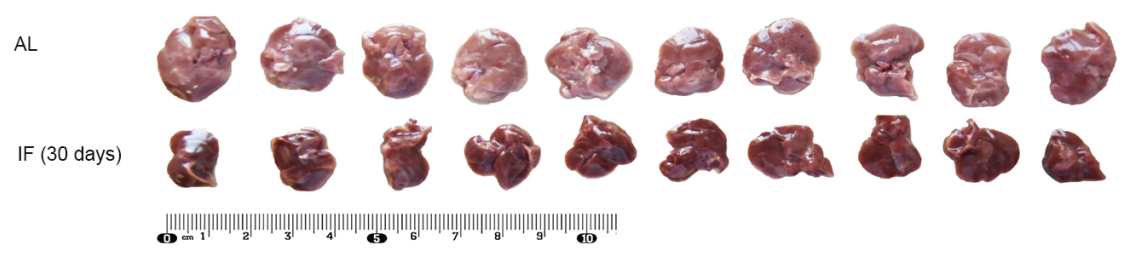

F

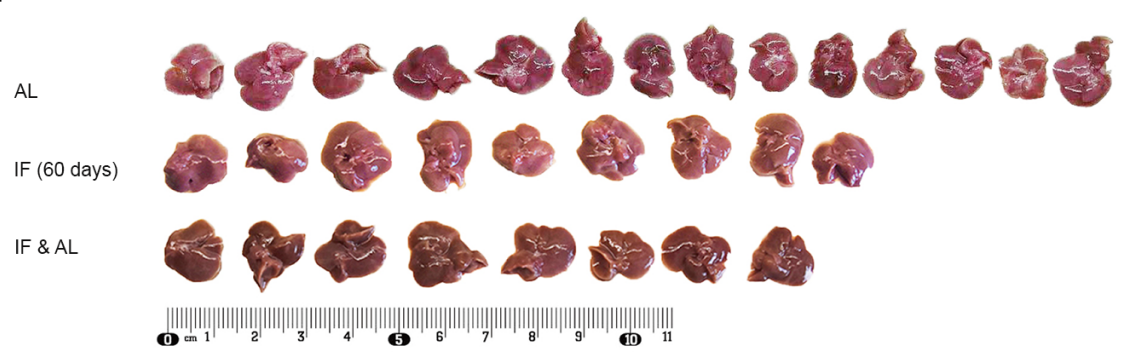

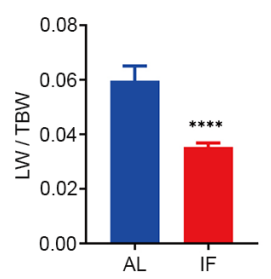

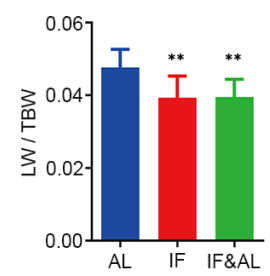

J

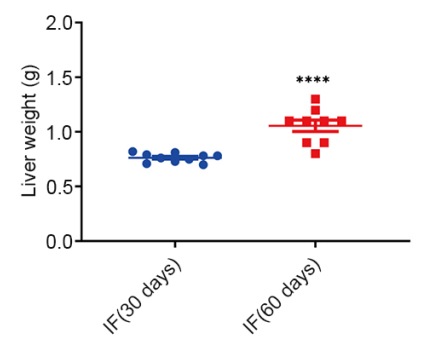

C

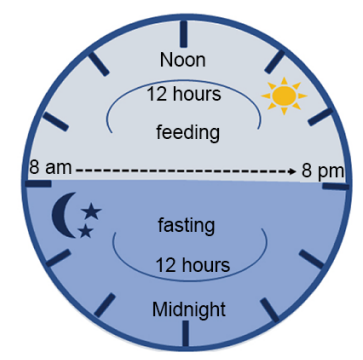

E

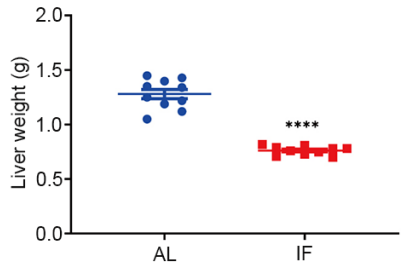

G

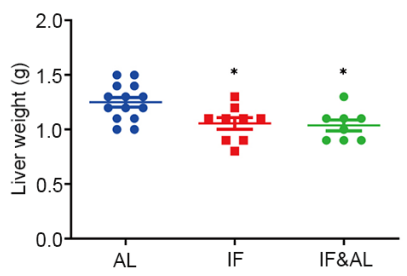

Figure 1. Effects of intermittent fasting on mouse livers. (A) Experimental design for the first experiment ( 30 days), including the schedule for the AL group $(\mathrm{n}=10)$ and IF group $(\mathrm{n}=10)$. In the AL group, mice had free access to food and water, while in the IF group, food and water were taken away for $12 \mathrm{~h}$ during the nighttime on each day. (B) Experimental design for the second experiment (60 days), including the AL group ( $\mathrm{n}=14)$, IF group ( $\mathrm{n}=9$ ) and IF followed by AL (IF\&AL) group ( $\mathrm{n}=8)$. In the AL group, mice had free access to food and water. In the IF group, food and water were taken away for $12 \mathrm{~h}$ during the nighttime on each day. In the IF\&AL group, the mice were given free access to food and water after one month of IF. (C) Schematic illustrating the experimental design for the timing of feeding and fasting. (D) Images of mouse livers from the first experiment. (E) Quantification of the liver weight in the first experiment. (F) Images of mouse livers from the first experiment. (G) Quantification of the liver weight in the second experiment. (H and I) Quantification of LW/TBW in $(\mathrm{H})$ the first experiment and (I) the second experiment. (J) Liver weight and (K) LW/TBW comparison between mice fasted for 30 and 60 days. Values are expressed as the mean \pm standard error of the mean. $\left.{ }^{*} \mathrm{P}<0.05,{ }^{* * *} \mathrm{P}<0.01,{ }^{* * * * *} \mathrm{P}<0.0001\right)$. IF, intermittent fasting; AL, ad libitum; LW/TBW, liver weight/total bodyweight.

metabolomics was performed by LC-MS/MS analysis. A total of 27 metabolite molecules related to energy metabolism (right column of Fig. 4B) were selected as targets. The principal component analysis provided clear clustering based on the diet patterns of the groups of mice (Fig. 4A). The distribution of the most significantly differentially expressed metabolites was visualized in a heatmap (Fig. 4B); this was used to separate the AL and the IF mice. A $>1.5$-fold increase was noted for 12 metabolite molecules in the IF mice compared to those in the AL mice (Fig. 5A-L) and the differences in Fig. 5F-I were particularly significant, While the expression of the other molecules did not exhibit any significant differences. The molecules exhibiting increased expression included nicotinamide adenine dinucleotide phosphate (NADP), adenosine triphosphate and succinate, whereas the main molecule exhibiting reduced expression was NADP. The majority of these metabolites are essentially involved in the citric acid cycle and oxidative phosphorylation. It was thus indicated that intermittent fasting enhanced liver metabolism, which may explain the reduction in the liver mass of these mice. 
Table I. Bodyweight and food intake of the mice.

\begin{tabular}{lcccccc}
\hline & \multicolumn{2}{c}{ 30-day experiment } & & \multicolumn{3}{c}{ 60-day experiment } \\
\cline { 2 - 3 } Item & $\mathrm{AL}(\mathrm{n}=10)$ & $\mathrm{IF}(\mathrm{n}=10)$ & & $\mathrm{AL}(\mathrm{n}=14)$ & $\mathrm{IF}(\mathrm{n}=9)$ & $\mathrm{IF \& AL}(\mathrm{n}=8)$ \\
\hline Bodyweight $(\mathrm{g})$ & & & & & \\
$\quad$ Beginning of experiment & $15.578 \pm 1.172$ & $14.890 \pm 0.853$ & & $21.764 \pm 1.381$ & $20.900 \pm 1.166$ & $20.613 \pm 1.629$ \\
End of experiment & $21.701 \pm 1.305$ & $21.610 \pm 1.187$ & & $26.193 \pm 1.680$ & $26.844 \pm 1.136$ & $26.457 \pm 1.779$ \\
Gain of weight $(\%)$ & 39.305 & 45.132 & & 20.350 & 28.440 & 28.351 \\
Food intake per day $(\mathrm{g})$ & & & & & & \\
Beginning of experiment & $3.667 \pm 0.343$ & $3.380 \pm 0.305^{\mathrm{a}}$ & & $2.729 \pm 0.580$ & $3.789 \pm 0.607^{\mathrm{b}}$ & $3.325 \pm 0.486$ \\
Middle of experiment & $3.770 \pm 0.393$ & $3.219 \pm 0.238^{\mathrm{c}}$ & & $3.221 \pm 0.691$ & $2.889 \pm 0.389$ & $2.763 \pm 0.277$ \\
End of experiment & $3.765 \pm 0.477$ & $2.721 \pm 0.393^{\mathrm{d}}$ & & $3.536 \pm 0.371$ & $2.433 \pm 0.424^{\mathrm{b}}$ & $2.625 \pm 0.358^{\mathrm{c}}$ \\
\hline
\end{tabular}

Beginning of experiment: 10th day (30 days experiment), 20th day (60 days experiment); Middle of experiment: 20th day (30 days experiment), 40th day (60 days experiment); End of experiment: 30th day (30 days experiment), 60th day (60 days experiment). ${ }^{\mathrm{a}} \mathrm{P}<0.05$, $30 \mathrm{AL}$ vs $30 \mathrm{days}$ IF group; ${ }^{\text {P}} \mathrm{P}<0.001,60 \mathrm{AL}$ vs 60 days IF group; ${ }^{\mathrm{c}} \mathrm{P}<0.01,30 \mathrm{AL}$ vs 30 days IF group or 60 days AL vs IF\&AL group; ${ }^{\mathrm{P}} \mathrm{P}<0.0001,30 \mathrm{AL}$ vs 30 days IF group. Values are expressed as the mean \pm SEM. AL, ad libitum group; IF, intermittent fasting group; IF\&AL, IF followed by AL group.

Table II. Bodyweight and food intake of the mice compared between 30 and 60 days of IF.

\begin{tabular}{lcc}
\hline Item & IF (30 days, $\mathrm{n}=10)$ & IF $(60$ days, $\mathrm{n}=9)$ \\
\hline Bodyweight $(\mathrm{g})$ & & $20.900 \pm 1.166^{\mathrm{a}}$ \\
Beginning of experiment & $14.890 \pm 0.853$ & $26.844 \pm 1.136^{\mathrm{a}}$ \\
End of experiment & $21.610 \pm 1.187$ & 28.440 \\
Gain of weight $(\%)$ & 45.132 & $3.789 \pm 0.607$ \\
Food intake per day $(\mathrm{g})$ & & $2.889 \pm 0.389$ \\
Beginning of experiment & $3.380 \pm 0.305$ & $2.433 \pm 0.424$ \\
Middle of experiment & $3.219 \pm 0.238$ & $2.721 \pm 0.393$ \\
End of experiment & & \\
\hline
\end{tabular}

Beginning of experiment: 10th day (30 days experiment), 20th day (60 days experiment); Middle of experiment: 20th day (30 days experiment), 40th day (60 days experiment); End of experiment: 30th day (30 days experiment), 60th day (60 days experiment). ${ }^{\text {a }}<0.0001,60$ days IF vs . 30 days IF group. Values are expressed as the mean \pm SEM. IF, intermittent fasting.

\section{Discussion}

Fasting, in particular intermittent fasting, has emerged as a health-promoting diet pattern, which is supported by evidence derived from both animal and human studies $(2,28,33,34)$. However, the patterns of intermittent fasting, depending on individual practice, vary tremendously. This poses major challenges in research with regard to experimental design and study of the most relevant intermittent fasting regimen. In the present study, Ramadan fasting was simulated in a mouse model involving deprivation of both food and water, similarly to several previous studies (35-38). Ramadan fasting has been practiced by over one billion Muslims for over 1,000 years and comprises daily fasting from dawn to dusk and eating without any restriction at night (4). The period of Ramadan fasting lasts for 29-30 days yearly (4). Previous studies have reported a modest weight loss by the end of Ramadan, whereas the mean weight loss was regained a few weeks after Ramadan $(39,40)$. However, other studies have reported weight gain following Ramadan fasting $(19,20)$. The present study simulated Ramadan fasting in mouse model. However, mice naturally feed and drink during the night with high level of physical activity, which is the opposite to humans. Considering that the feeding habits of mice are different compared to those of humans due to nocturnal circadian rhythms, our study in mouse model resembled Ramadan fasting to a certain extent. The results indicated that daily intermittent fasting for $12 \mathrm{~h}$ at night for 1 month reduced blood glucose levels and enhanced liver metabolism, although this did not affect the bodyweight of the mice. However, it is important to note that the mouse studies have limitations that translate to humans, including food composition and environmental diversity.

Various studies have previously focused on the ability of the diet to prevent disease development and progression. A novel theorem has emerged which suggests that the time period of eating is also important. It has been indicated that the timing of food intake affects metabolic health and cancer development (41-43). A large prospective cohort study of 2,413 patients with breast cancer 

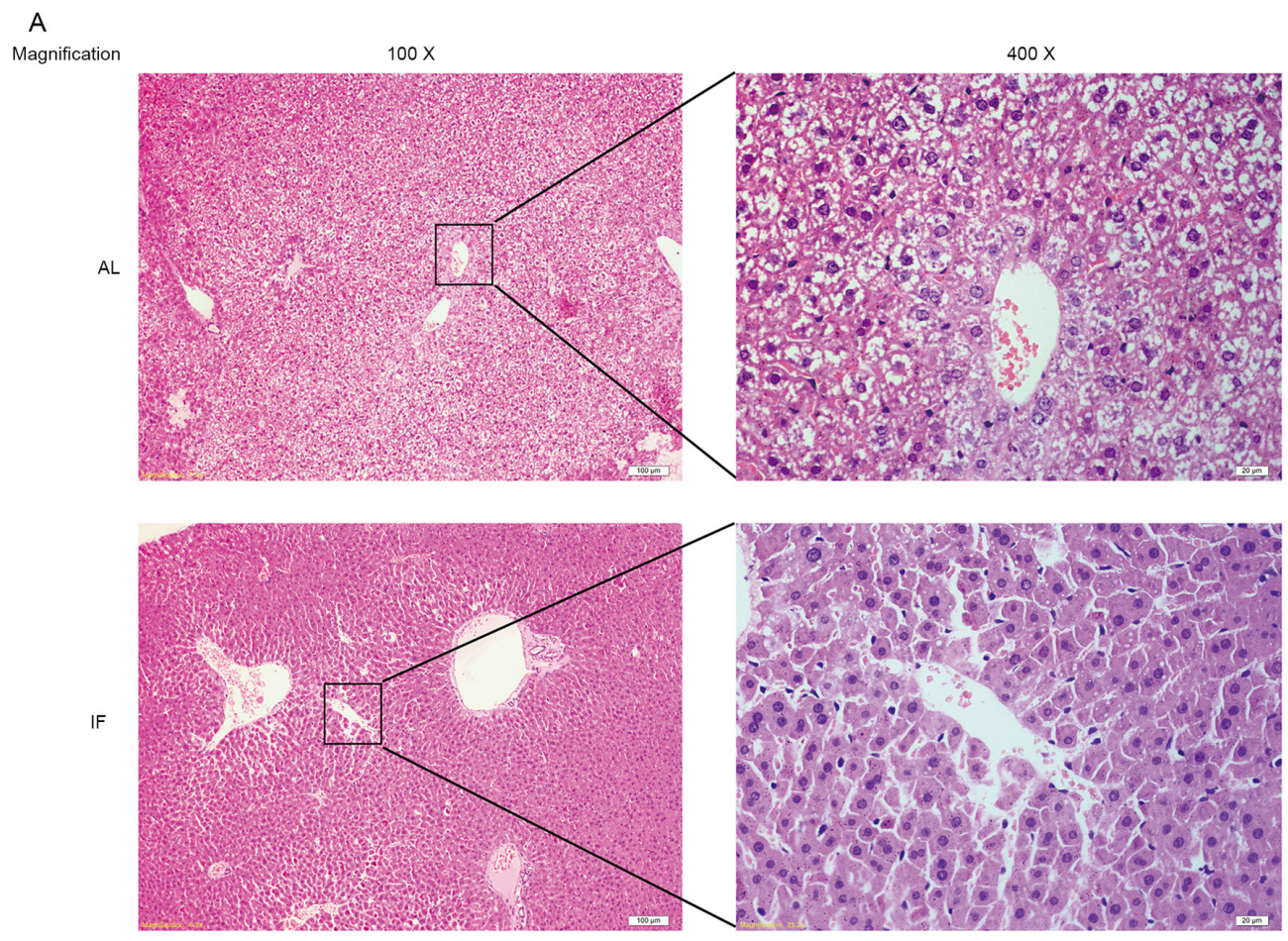

B
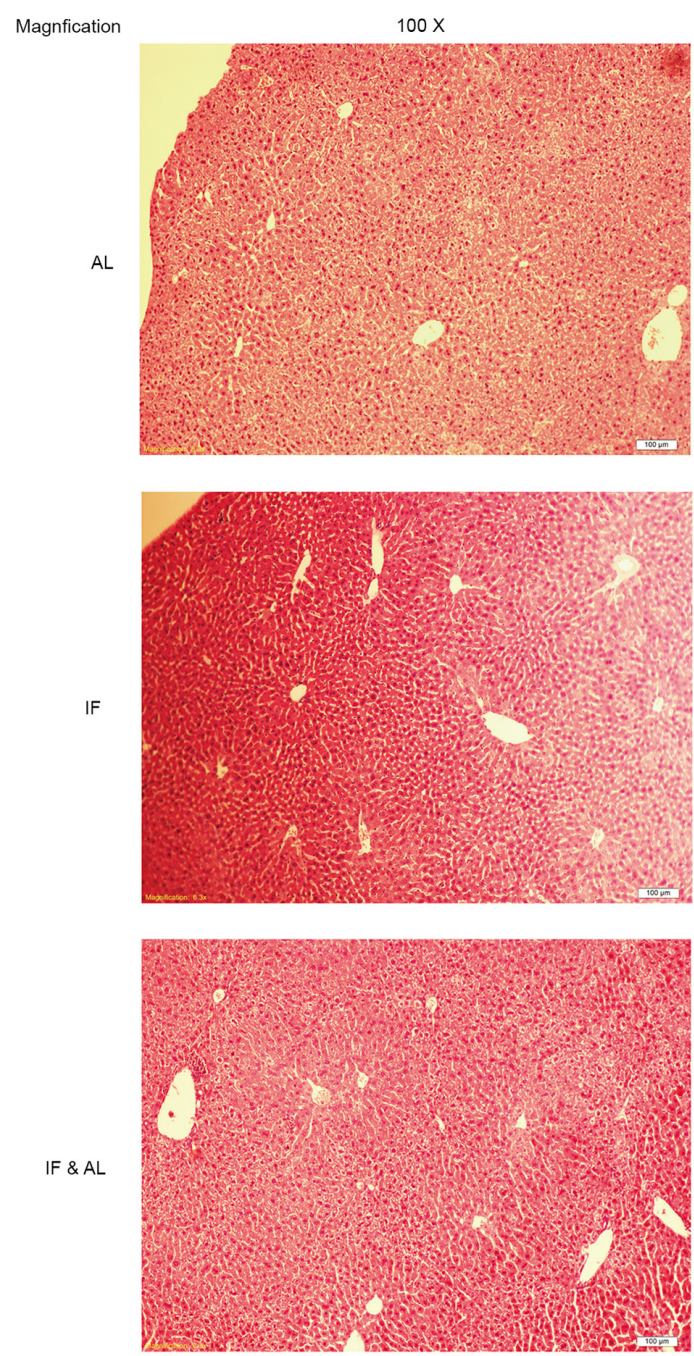
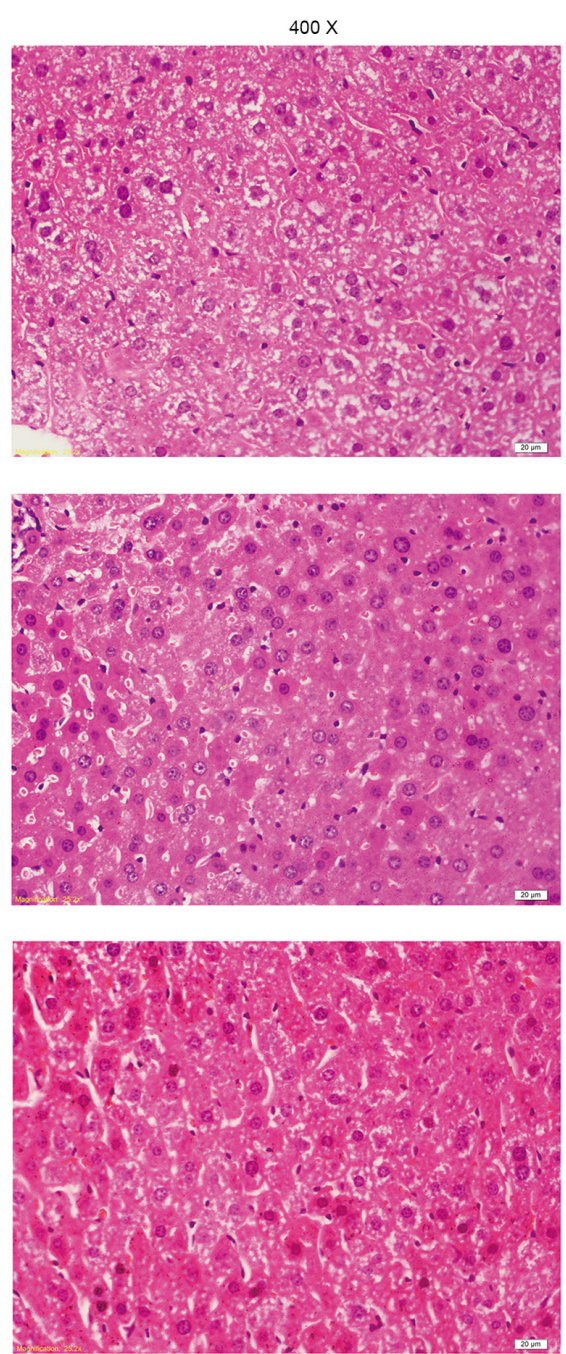

Figure 2. Histological analysis of liver morphology. (A) First experiment (30 days); AL and IF groups; (B) Second experiment (60 days); AL, IF and IF\&AL groups (H\&E staining; magnification, x100 in the left and x400 in the right panel; scale bars, $100 \mu \mathrm{m}$ in the left and $20 \mu \mathrm{m}$ in the right panel). IF, intermittent fasting; AL, ad libitum; IF\&AL, intermittent fasting followed by ad libitum. 
A

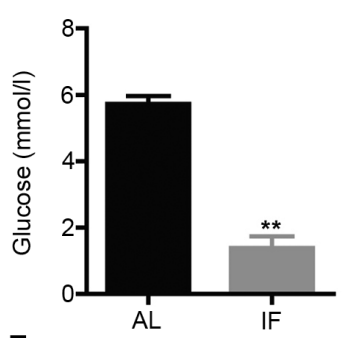

E

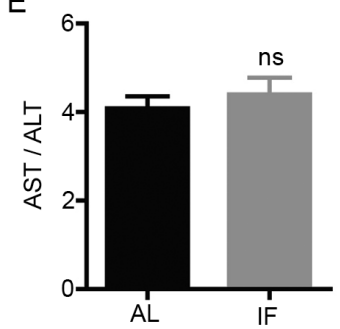

I

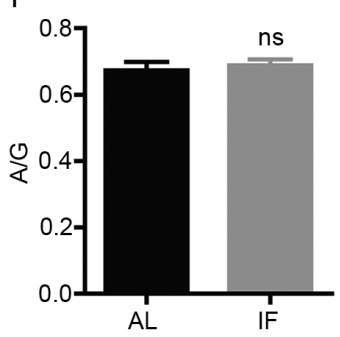

B

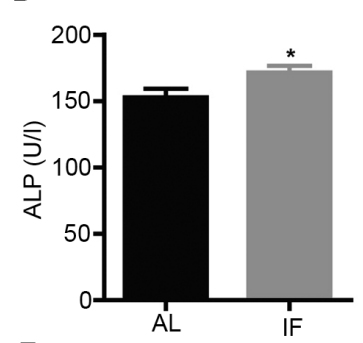

$\mathrm{F}$

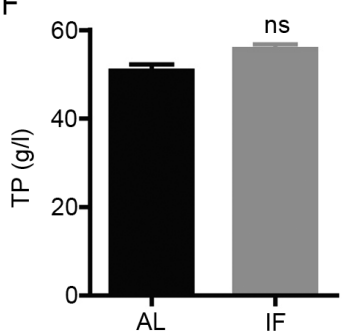

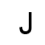

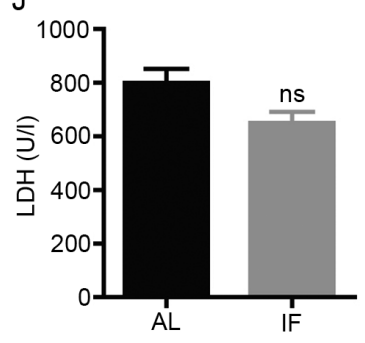

C

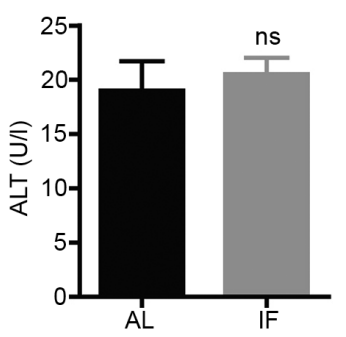

G

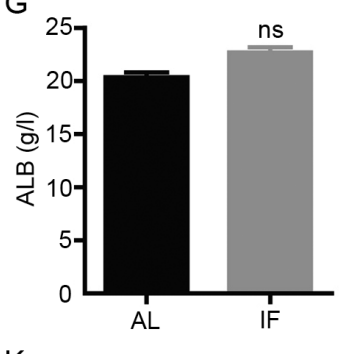

$\mathrm{K}$

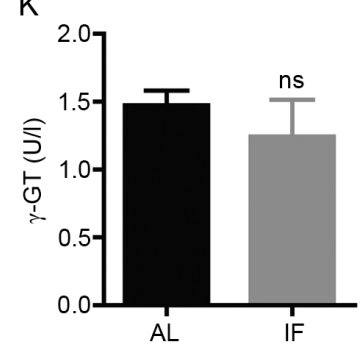

D

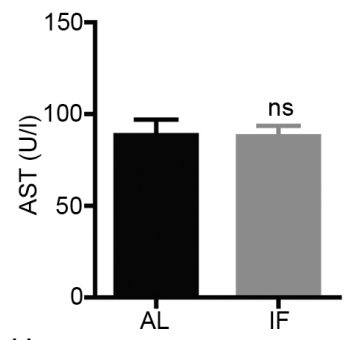

$\mathrm{H}$

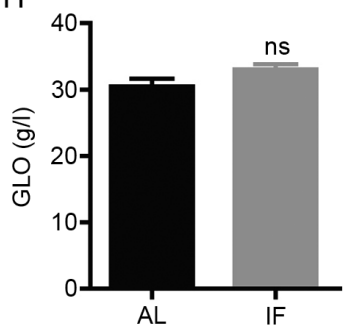

$\mathrm{AL}$
$\mathrm{IF}$

Figure 3. Levels of blood biochemical markers at 30 days. (A) Blood glucose; (B) ALP; (C) ALT; (D) AST; (E) AST/ALT; (F) TP; (G) ALB; (H) GLO; (I) A/G; (J) LDH; and (K) $\gamma$-GT. Values are expressed as the mean \pm standard error of the mean $(\mathrm{n}=7)$. " $\mathrm{P}<0.05,{ }^{* * *} \mathrm{P}<0.01$; ns, no significance. IF, intermittent fasting; AL, ad libitum; ALP, alkaline phosphatase; ALT, alanine aminotransferase; ALB, albumin; AST, aspartate aminotransferase; A/G, ALB/GLO; GLO, globulin; $\gamma$-GT, $\gamma$-glutamyl transpeptidase; LDH, lactate dehydrogenase; TP, total protein.

A

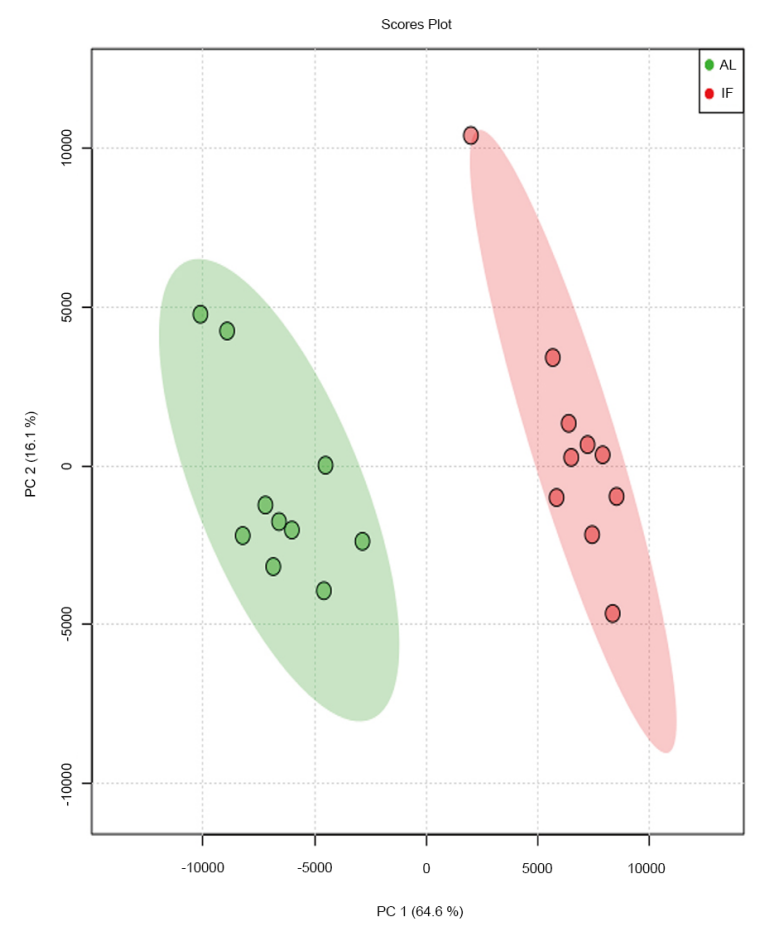

B

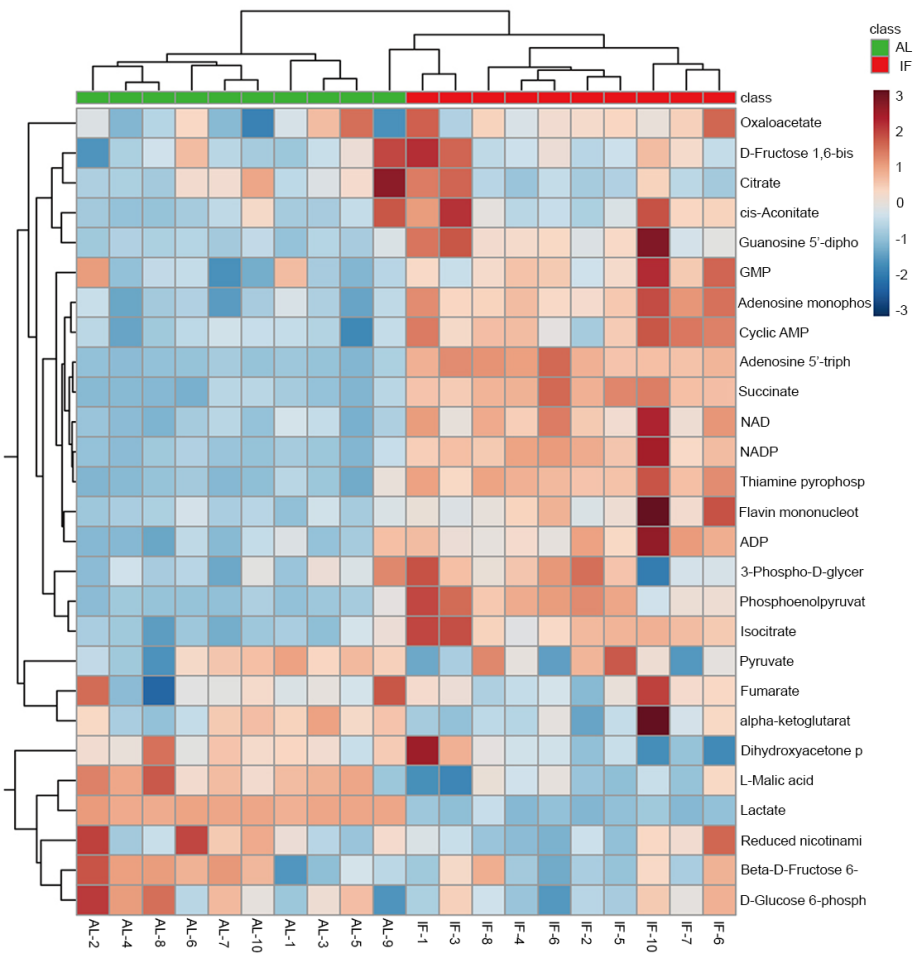

Figure 4. Targeted metabolomics analysis of the mouse liver. (A) PC analysis plots were generated based on diet patterns and the difference was identified. (B) The 27 metabolites were analyzed by using liquid chromatography tandem mass spectrometry and the heatmap indicated differences between the IF and AL mice at 30 days. PC, principal component; IF, intermittent fasting; AL, ad libitum; NADP, nicotinamide adenine dinucleotide phosphate; GMP, guanosine monophosphate; ADP, adenosine diphosphate. 


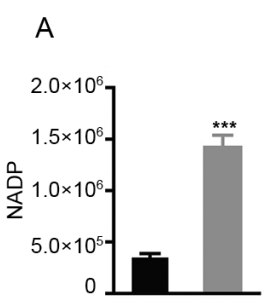

B

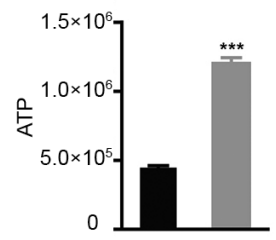

$\mathrm{H}$
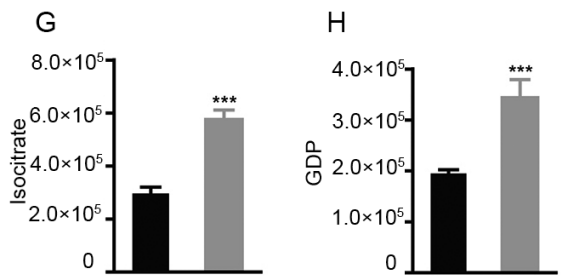

C

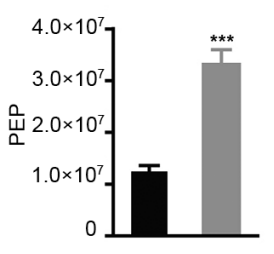

I

$\mathrm{D}$

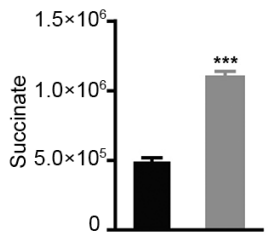

$\mathrm{J}$
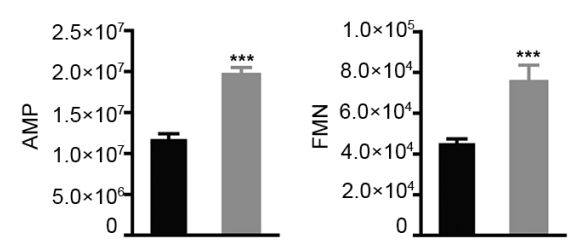

E

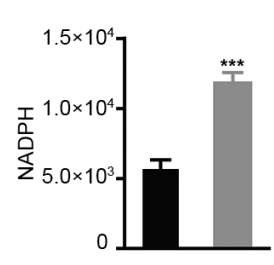

$\mathrm{F}$

$\mathrm{AL}$
$\mathrm{IF}$

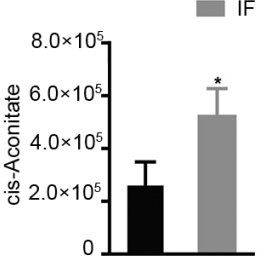

K

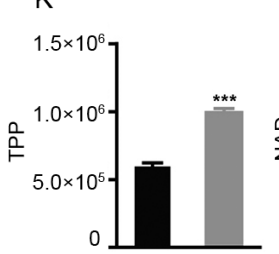

L

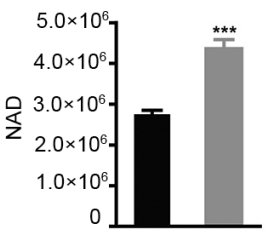

Figure 5. Among the 27 metabolites analyzed, 12 were significantly increased by intermittent fasting for 30 days. (A) NADP; (B) ATP; (C) PEP; (D) Succinate; (E) NADPH; (F) cis-aconitate; (G) isocitrate; (H) GDP; (I) AMP; (J) FMN; (K) TPP; (L) NAD. Values are expressed as the mean \pm standard error of the mean $(\mathrm{n}=10) .{ }^{*} \mathrm{P}<0.05,{ }^{* * * *} \mathrm{P}<0.001$. IF, intermittent fasting; AL, ad libitum; AMP, adenosine monophosphate; ATP, adenosine triphosphate; FMN, flavin mononucleotide; GDP, guanosine diphosphate; NADP, nicotinamide adenine dinucleotide phosphate; NADPH, reduced NADP; PEP, phosphoenolpyruvate; TPP, thiamine pyrophosphate.

reported that a short duration of fasting in the night $(<13 \mathrm{~h}$ per night) was associated with a $36 \%$ increased risk of cancer recurrence (44). Therefore, erratic feeding behaviors and/or circadian rhythms may have detrimental health consequences (45-47).

Disruption of the circadian rhythm has been identified as a common risk factor for obesity, metabolic disorders, non-alcoholic fatty liver diseases and liver cancer (48-51). At least $10 \%$ of the liver transcriptome exhibits rhythmic gene expression, suggesting that the circadian clock regulates a large number of hepatic genes (52). It has been demonstrated that chronic circadian disruption promotes weight gain and hepatic lipid storage in mice $(53,54)$. This may be the reason for the loss of liver mass and the slight bodyweight gain in the mouse model used in the present study.

Under physiological conditions, the liver serves as the major organ for supplying energy to the body. It has been reported that 12-24 h of fasting results in depletion of hepatic glycogen, accompanied by a switch to a metabolic mode in which non-hepatic glucose, fat-derived ketone bodies and free fatty acids are used as energy sources (33). This suggests that intermittent energy deprivation is capable of improving metabolic health. However, the underlying mechanisms through which intermittent energy restrictions improve the health status have remained largely elusive. In the present study, a number of metabolites were identified that exhibited significant elevation in their expression levels in liver samples from mice of the IF group. These metabolites are essential components of the citric acid cycle, oxidative phosphorylation and glycolysis cascades, indicating the induction of liver metabolism. This may provide a mechanistic explanation for the effects of intermittent fasting on liver physiology. Although the metabolic consequences of intermittent fasting are complex and the mechanisms by which this process benefits metabolic regulation remain elusive, previous studies on the molecular changes occurring in the liver have provided potential non-pharmacological approaches to improving the health status in the general population $(55,56)$.
Previous studies on intermittent fasting in humans mainly focused on the therapeutic potential for different diseases, such as cardiovascular disorders, obesity and diabetes (57-59). The present study aimed to explore the physiological and biochemical changes occurring in the liver following intermittent fasting in healthy mice. A fasting pattern was adopted, which simulated Ramadan fasting to a certain extent. Therefore, the results may provide an understanding of the physiological effects of intermittent fasting on the general population. However, the mechanisms by which intermittent fasting provides therapeutic benefits require further investigation using specific disease models. A limitation of the present study was that liver metabolomics were only performed for the 30-day fasting group, mainly due to financial limitations. It would be worthwhile to perform this assay for the 60-day fasting group in future studies.

In conclusion, the present study indicated that intermittent fasting reduces liver weight and rewires liver metabolism in mice. This may partially contribute to the health benefits of fasting and provides approaches for therapeutic intervention in chronic diseases, such as non-alcoholic fatty liver disease. However, the mechanisms through which intermittent fasting regulates liver metabolism remain to be further investigated.

\section{Acknowledgements}

Not applicable.

\section{Funding}

The present study was supported by the National Natural Science Foundation of China (grant no. 81972281); the Program for Changjiang Scholars and Innovative Research Teams in University of the Ministry of Education, China (grant no. IRT_17R88) Medical Scientific Research, the foundation of Guangdong Province (grant no. A2015297). 


\section{Availability of data and materials}

The datasets used and/or analyzed during the current study are available from the corresponding author on reasonable request.

\section{Authors' contributions}

JM, YW, LL and QS performed the experiments. JM and YC analyzed the data and wrote the manuscript. WA, LG, ZM, $\mathrm{ZQ}, \mathrm{QP}$ and $\mathrm{KC}$ designed the project and discussed the results. $\mathrm{QP}$ and $\mathrm{KC}$ supervised the research and edited the manuscript. $\mathrm{JM}$ and $\mathrm{KC}$ confirm the authenticity of all the raw data. All authors read and approved the final manuscript.

\section{Ethics approval and consent to participate}

The animal study was performed with the approval of the Laboratory Animal Ethics Committee of Northwest Minzu University (Lanzhou, China).

\section{Patient consent for publication}

Not applicable.

\section{Competing interests}

The authors declare that they have no competing interests.

\section{References}

1. de Cabo R, Carmona-Gutierrez D, Bernier M, Hall MN and Madeo F: The search for antiaging interventions: From elixirs to fasting regimens. Cell 157: 1515-1526, 2014.

2. Longo VD and Panda S: Fasting, circadian rhythms, and time-restricted feeding in healthy lifespan. Cell Metab 23: 1048-1059, 2016.

3. Mitchell SJ, Bernier M, Mattison JA, Aon MA, Kaiser TA, Anson RM, Ikeno Y, Anderson RM, Ingram DK and de Cabo R: Daily fasting improves health and survival in male mice independent of diet composition and calories. Cell Metab 29: 221-228.e3, 2019.

4. Lessan $\mathrm{N}$ and Ali T: Energy metabolism and intermittent fasting: The Ramadan perspective. Nutrients 11: 1192, 2019.

5. Patterson RE, Laughlin GA, LaCroix AZ, Hartman SJ, Natarajan L, Senger CM, Martínez ME, Villaseñor A, Sears DD, Marinac CR and Gallo LC: Intermittent fasting and human metabolic health. J Acad Nutr Diet 115: 1203-1212, 2015.

6. Harney DJ, Hutchison AT, Hatchwell L, Humphrey SJ, James DE, Hocking S, Heilbronn LK and Larance M: Proteomic analysis of human plasma during intermittent fasting. J Proteome Res 18: 2228-2240, 2019.

7. Patterson RE and Sears DD: Metabolic effects of intermittent fasting. Annu Rev Nutr 37: 371-393, 2017.

8. Ahmad S and Chowdhury TA: Fasting during Ramadan in people with chronic kidney disease: A review of the literature. Ther Adv Endocrinol Metab 10: 2042018819889019, 2019.

9. Weindruch R and Sohal RS: Seminars in medicine of the Beth Israel deaconess medical center. Caloric intake and aging. N Engl J Med 337: 986-994, 1997.

10. Baumeier C, Kaiser D, Heeren J, Scheja L, John C, Weise C, Eravci M, Lagerpusch M, Schulze G, Joost HG, et al: Caloric restriction and intermittent fasting alter hepatic lipid droplet proteome and diacylglycerol species and prevent diabetes in NZO mice. Biochim Biophys Acta 1851: 566-576, 2015.

11. Belkacemi L, Selselet-Attou G, Bulur N, Louchami K, Sener A and Malaisse WJ: Intermittent fasting modulation of the diabetic syndrome in sand rats. III. Post-mortem investigations. Int J Mol Med 27: 95-102, 2011.
12. Horne BD, Muhlestein JB and Anderson JL: Health effects of intermittent fasting: Hormesis or harm? A systematic review. Am J Clin Nutr 102: 464-470, 2015.

13. Jane L, Atkinson G, Jaime V, Hamilton S, Waller G and Harrison S: Intermittent fasting interventions for the treatment of overweight and obesity in adults aged 18 years and over: A systematic review protocol. JBI Database System Rev Implement Rep 13: 60-68, 2015.

14. Nair KS, Woolf PD, Welle SL and Matthews DE: Leucine, glucose, and energy metabolism after 3 days of fasting in healthy human subjects. Am J Clin Nutr 46: 557-562, 1987.

15. Müller MJ, Enderle J, Pourhassan M, Braun W, Eggeling B, Lagerpusch M, Glüer CC, Kehayias JJ, Kiosz D and Bosy-Westphal A: Metabolic adaptation to caloric restriction and subsequent refeeding: The minnesota starvation experiment revisited. Am J Clin Nutr 102: 807-819, 2015.

16. Mansell PI and Macdonald IA: The effect of starvation on insulin-induced glucose disposal and thermogenesis in humans. Metabolism 39: 502-510, 1990.

17. Webber J and Macdonald IA: The cardiovascular, metabolic and hormonal changes accompanying acute starvation in men and women. Br J Nutr 71: 437-447, 1994.

18. Haouari M, Haouari-Oukerro F, Sfaxi A, Ben Rayana MC, Kâabachi N and Mbazâa A: How Ramadan fasting affects caloric consumption, body weight, and circadian evolution of cortisol serum levels in young, healthy male volunteers. Horm Metab Res 40: 575-577, 2008.

19. Kul S, Savaş E, ÖztürkZA and Karadă̆ G: Does Ramadan fasting alter body weight and blood lipids and fasting blood glucose in a healthy population? A meta-analysis. J Relig Health 53: 929-942, 2014.

20. Sadeghirad B, Motaghipisheh S, Kolahdooz F, Zahedi MJ and Haghdoost AA: Islamic fasting and weight loss: A systematic review and meta-analysis. Public Health Nutr 17: 396-406, 2014.

21. Charlton MR: Protein metabolism and liver disease. Baillieres Clin Endocrinol Metab 10: 617-635, 1996.

22. Lawrence YA, Guard BC, Steiner JM, Suchodolski JS and Lidbury JA: Untargeted metabolomic profiling of urine from healthy dogs and dogs with chronic hepatic disease. PLoS One 14: e0217797, 2019.

23. Besse-Patin A, Jeromson S, Levesque-Damphousse P, Secco B, Laplante $\mathrm{M}$ and Estall JL: PGC1A regulates the IRS1:IRS2 ratio during fasting to influence hepatic metabolism downstream of insulin. Proc Natl Acad Sci USA 116: 4285-4290, 2019.

24. Moore MC, Coate KC, Winnick JJ, An Z and Cherrington AD: Regulation of hepatic glucose uptake and storage in vivo. Adv Nutr 3: 286-294, 2012.

25. Ramnanan CJ, Edgerton DS, Kraft G and Cherrington AD: Physiologic action of glucagon on liver glucose metabolism. Diabetes Obes Metab 13 (Suppl 1): S118-S125, 2011.

26. Gibney MJ, Walsh M, Brennan L, Roche HM, German B and van Ommen B: Metabolomics in human nutrition: Opportunities and challenges. Am J Clin Nutr 82: 497-503, 2005.

27. Hollywood K, Brison DR and Goodacre R: Metabolomics: Current technologies and future trends. Proteomics 6: 4716-4723, 2006.

28. de Cabo R and Mattson MP: Effects of intermittent fasting on health, aging, and disease. N Engl J Med 381: 2541-2551, 2019.

29. Golbidi S, Daiber A, Korac B, Li H, Essop MF and Laher I: Health benefits of fasting and caloric restriction. Curr Diab Rep 17: 123, 2017.

30. Johnson EL: Seizures and epilepsy. Med Clin North Am 103: 309-324, 2019.

31. Guo ZV, Hires SA, Li N, O'Connor DH, Komiyama T, Ophir E, Huber D, Bonardi C, Morandell K, Gutnisky D, et al: Procedures for behavioral experiments in head-fixed mice. PLoS One 9: e88678, 2014

32. Tucci V, Hardy A and Nolan PM: A comparison of physiological and behavioural parameters in C57BL/6J mice undergoing food or water restriction regimes. Behav Brain Res 173: 22-29, 2006.

33. Longo VD and Mattson MP: Fasting: Molecular mechanisms and clinical applications. Cell Metab 19: 181-192, 2014.

34. Wegman MP, Guo MH, Bennion DM, Shankar MN, Chrzanowski SM, Goldberg LA, Xu J, Williams TA, Lu X, Hsu SI, et al: Practicality of intermittent fasting in humans and its effect on oxidative stress and genes related to aging and metabolism. Rejuvenation Res 18: 162-172, 2015.

35. Manzanero S,Erion JR,Santro T, SteynFJ, Chen C,ArumugamTV and Stranahan AM: Intermittent fasting attenuates increases in neurogenesis after ischemia and reperfusion and improves recovery. J Cereb Blood Flow Metab 34: 897-905, 2014. 
36. Faroog N, Priyamvada S, Arivarasu NA, Salim S, Khan F and Yusufi AN: Influence of Ramadan-type fasting on enzymes of carbohydrate metabolism and brush border membrane in small intestine and liver of rat used as a model. Br J Nutr 96: 1087-1094, 2006.

37. Mohany M, Ashton N, Harrath AH, Nyengaard JR, Alomar SY and Alwasel S: A new model for fetal programming: Maternal Ramadan-type fasting programs nephrogenesis. J Dev Orig Health Dis 9: 287-298, 2018

38. Salim S, Farooq N, Priyamvada S, Asghar M, Khundmiri SJ, Khan S, Khan F and Yusufi AN: Influence of Ramadan-type fasting on carbohydrate metabolism, brush border membrane enzymes and phosphate transport in rat kidney used as a model. Br J Nutr 98: 984-990, 2007.

39. Fernando HA, Zibellini J, Harris RA, Seimon RV and Sainsbury A: Effect of Ramadan fasting on weight and body composition in healthy non-athlete adults: A systematic review and meta-analysis. Nutrients 11: 478, 2019.

40. Sethi BK and Nagesh VS: Weight management in Ramadan. J Pak Med Assoc 65 (5 Suppl 1): S54-S56, 2015.

41. Hutchison AT and Heilbronn LK: Metabolic impacts of altering meal frequency and timing-does when we eat matter? Biochimie 124: 187-197, 2016.

42. Mattson MP, Allison DB, Fontana L, Harvie M, Longo VD, Malaisse WJ, Mosley M, Notterpek L, Ravussin E, Scheer FA, et al: Meal frequency and timing in health and disease. Proc Natl Acad Sci USA 111: 16647-16653, 2014.

43. Marinac CR, Natarajan L, Sears DD, Gallo LC, Hartman SJ, Arredondo E and Patterson RE: Prolonged nightly fasting and breast cancer risk: Findings from NHANES (2009-2010). Cancer Epidemiol Biomarkers Prev 24: 783-789, 2015.

44. Marinac CR, Nelson SH, Breen CI, Hartman SJ, Natarajan L, Pierce JP, Flatt SW, Sears DD and Patterson RE: Prolonged nightly fasting and breast cancer prognosis. JAMA Oncol 2: 1049-1055, 2016

45. Gill S and Panda S: A smartphone App reveals erratic diurnal eating patterns in humans that can be modulated for health benefits. Cell Metab 22: 789-798, 2015.

46. Manoogian ENC and Panda S: Circadian rhythms, time-restricted feeding, and healthy aging. Ageing Res Rev 39: 59-67, 2017.

47. Jain Gupta $\mathrm{N}$ and Khare A: Disruption in daily eating-fasting and activity-rest cycles in Indian adolescents attending school. PLoS One 15: e0227002, 2020

48. Chen K, Ma J, Jia X, Ai W, Ma Z and Pan Q: Advancing the understanding of NAFLD to hepatocellular carcinoma development: From experimental models to humans. Biochim Biophys Acta Rev Cancer 1871: 117-125, 2019.
49. Fu L and Kettner NM: The circadian clock in cancer development and therapy. Prog Mol Biol Transl Sci 119: 221-282, 2013.

50. Kim CW, Yun KE, Jung HS, Chang Y, Choi ES, Kwon MJ, Lee EH, Woo EJ, Kim NH, Shin H and Ryu S: Sleep duration and quality in relation to non-alcoholic fatty liver disease in middle-aged workers and their spouses. J Hepatol 59: 351-357, 2013.

51. Maury E: Off the clock: From circadian disruption to metabolic disease. Int J Mol Sci 20: 1597, 2019.

52. Hunter AL and Ray DW: Circadian clock regulation of hepatic energy metabolism regulatory circuits. Biology (Basel) 8: 79, 2019.

53. Christie S, Vincent AD, Li H, Frisby CL, Kentish SJ, O'Rielly R, Wittert GA and Page AJ: A rotating light cycle promotes weight gain and hepatic lipid storage in mice. Am J Physiol Gastrointest Liver Physiol 315: G932-G942, 2018.

54. Fleet T, Stashi E, Zhu B, Rajapakshe K, Marcelo KL, Kettner NM, Gorman BK, Coarfa C, Fu L, O'Malley BW and York B: Genetic and environmental models of circadian disruption link SRC-2 function to hepatic pathology. J Biol Rhythms 31: 443-460, 2016.

55. Bertholdt L, Gudiksen A, Jessen H and Pilegaard H: Impact of skeletal muscle IL- 6 on regulation of liver and adipose tissue metabolism during fasting. Pflugers Arch 470: 1597-1613, 2018.

56. Marinho TS, Ornellas F, Barbosa-da-Silva S Mandarim-de-Lacerda CA and Aguila MB: Beneficial effects of intermittent fasting on steatosis and inflammation of the liver in mice fed a high-fat or a high-fructose diet. Nutrition 65: 103-112, 2019.

57. Johnstone A: Fasting for weight loss: An effective strategy or latest dieting trend? Int J Obes (Lond) 39: 727-733, 2015.

58. Barnosky AR, Hoddy KK, Unterman TG and Varady KA: Intermittent fasting vs daily calorie restriction for type 2 diabetes prevention: A review of human findings. Transl Res 164: 302-311, 2014.

59. Carter S, Clifton PM and Keogh JB: The effects of intermittent compared to continuous energy restriction on glycaemic control in type 2 diabetes; a pragmatic pilot trial. Diabetes Res Clin Pract 122: 106-112, 2016.

This work is licensed under a Creative Commons Attribution-NonCommercial-NoDerivatives 4.0 International (CC BY-NC-ND 4.0) License. 